\title{
A Novel Open-Loop Speed Estimation Technique for Low-Cost IM Drives
}

\author{
Silverio Bolognani \\ University of Padova \\ Department of Electrical Engineering \\ Via Gradenigo 6/A \\ 35131 Padova - Italy
}

\author{
Luca Peretti and Mauro Zigliotto \\ University of Padova \\ Department of Industrial Technology and Management \\ Stradella San Nicola 3 \\ 36100 Vicenza - Italy
}

\begin{abstract}
In the last decades, several schemes have been proposed for speed-sensorless control of Induction Motor (IM) drives. Promising approaches are closed-loop observers, as Model Reference Adaptive Systems (MRAS) and Kalman filters. Recently, open-loop estimators have aroused lively interest because of their simplicity and low-cost profile. As known, open-loop algorithms use only motor equations to derive the speed estimation, whose accuracy turns out to be strictly related to the motor model and its parameters. Also the digital implementation of differentiator/integrator blocks plays a crucial role, and sometimes it reduces the applicability of open loop estimator to a limited speed range. This paper presents a novel open-loop speed estimate, with enhanced performances in terms of both speed range and robustness against parameters uncertainty. The task is accomplished with a hybrid mathematical model, which maintains a low-cost profile suitable for the industrial scenario. Extensive experimental results conclude the paper.
\end{abstract}

\section{INTRODUCTION}

Sensorless control of Induction Motor (IM) drives have been profusely investigated during the last decades. The manifold proposals present in the literature can be classified into two main groups. The first one is composed by closed-loop observers, in which the speed guess is forced to track a null error between estimated and real values of a measured variable (for example, the stator current). The category comprises Luenberger Observers [1] and Kalman Filters, which are burdensome because of the heavy mathematical approach and their tuning difficulties [2], [3]. Also Model Reference Adaptive Systems (MRAS) are closed-loop algorithms, inherently nonlinear in both the error processed by the adaptive controller and in the mathematical model modified by the feedback contribute [4], [5]. MRAS-based algorithms claim to be an interesting solution for industrial applications, since their implementation is simple in spite of their non-linearity, and the stability issues have been well deepened already.

The second group of sensorless methods is composed by open-loop algorithms. Opposite to closed-loop approach, the estimated speed is directly obtained from the machine equations, by measuring both phase voltages and currents. In principle, open-loop approaches tend to be simpler than closed-loop counterparts, mainly because they often use reduced machine models for speed estimation and they have not closed loop regulators.
The major disadvantage of the open-loop approach is represented by its sensitivity to parameters values. The use of reduced models, combined with the absence of a feedback correction, leads to potentially poor performances in presence of parameter inaccuracies, which may even cause instability.

Another key-issue is represented by the implementation of integral and differential blocks. As known, any DC offset at integrator input causes output saturation. As well, a differentiator output is always affected by the noise superimposed to its input. These negative effects, which are amplified in digital implementation, can negatively bias the whole performance of an open-loop sensorless system.

For the aforementioned reasons, open-loop algorithms are often restricted to low-cost systems, with limited speed range and low dynamic requirements. However, it will be shown that a proper mathematical hybrid model can sensibly improve both the mentioned features. In this paper, the inherent simplicity of open-loop method is preserved, resulting in a low-cost implementation, suitable for the targeted industrial scenario.

The novel open-loop solution is derived from an existing method, reported in [6]. The modification introduced in the mathematical model gives superior performances with respect to the original one. Theoretical background and accurate sensitivity analysis, as well as a complete set of experimental results are reported in the paper, demonstrating the effectiveness of the proposal.

\section{THE OPEN-LOOP ESTIMATOR}

The proposed open-loop estimator is based on the fourparameters equivalent circuit of a generic IM, depicted in Figure 1.

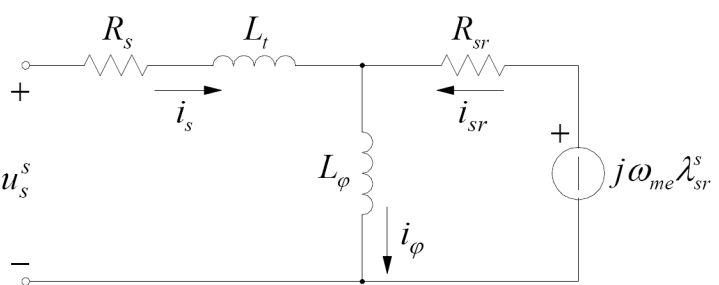

Figure 1. Four-parameters electrical equivalent circuit of a generic IM

In Figure 1 the parameters are the stator resistance $R_{s}$, the 
transient inductance $L_{t}$, the magnetising inductance $L_{\varphi}$ and the rotor resistance referred to the stator $R_{s r}$. The variables $\boldsymbol{i}_{\varphi}^{s}, \boldsymbol{i}_{s r}^{s}$ and $\boldsymbol{\lambda}_{s r}^{s}$ are the magnetising current vector, the rotor current vector referred to the stator, and the rotor flux linkage vector referred to the stator, respectively. The following relationships hold between $\boldsymbol{i}_{s r}^{s}, \boldsymbol{\lambda}_{s r}^{s}$, the stator current vector $\boldsymbol{i}_{s}^{s}$ and the stator flux linkage vector $\lambda_{s}^{s}$ :

$$
\begin{aligned}
\boldsymbol{i}_{s r}^{s} & =\frac{\boldsymbol{\lambda}_{s r}^{s}}{L_{\varphi}}-\boldsymbol{i}_{s}^{s} \\
\boldsymbol{\lambda}_{s}^{s} & =L_{t} \boldsymbol{i}_{s}^{s}+\boldsymbol{\lambda}_{s r}^{s}
\end{aligned}
$$

If the stator flux $\lambda_{s}^{s}$ is calculated from the stator model as the time integral of the voltage $\boldsymbol{u}_{s}^{s}$ [7], from (2) it follows:

$$
\boldsymbol{\lambda}_{s r}^{s}=\int_{-\infty}^{t}\left(\boldsymbol{u}_{s}^{s}-R_{s} \boldsymbol{i}_{s}^{s}\right) d t-L_{t} \boldsymbol{i}_{s}^{s}
$$

The mathematical model of the open-loop sensorless estimation can be derived from the right (rotor) side of fourparameters circuit of Figure 1. One can write

$$
\mathbf{0}=R_{s r} i_{s r}^{s}+\frac{d \boldsymbol{\lambda}_{s r}^{s}}{d t}-j \omega_{m e} \boldsymbol{\lambda}_{s r}^{s}
$$

Substituting (1) into (4) gives

$$
\frac{d \boldsymbol{\lambda}_{s r}^{s}}{d t}=\left(j \omega_{m e}-\frac{R_{s r}}{L_{\varphi}}\right) \boldsymbol{\lambda}_{s r}^{s}+R_{s r} i_{s}^{s}
$$

The expression (5) can be written in the bi-dimensional stationary reference frame $\alpha-\beta$ :

$$
\left\{\begin{array}{l}
\frac{d \lambda_{s r \alpha}^{s}}{d t}+\frac{R_{s r}}{L_{\varphi}} \lambda_{s r \alpha}^{s}=-\omega_{m e} \lambda_{s r \beta}^{s}+R_{s r} i_{s \alpha}^{s} \\
\frac{d \lambda_{s r \beta}^{s}}{d t}+\frac{R_{s r}}{L_{\varphi}} \lambda_{s r \beta}^{s}=\omega_{m e} \lambda_{s r \alpha}^{s}+R_{s r} i_{s \beta}^{s}
\end{array}\right.
$$

Solving the first of (6) for $\omega_{m e}$ gives

$$
\omega_{m e, 1}=-\frac{\left(-R_{s r} i_{s \alpha}^{s}+\frac{d \lambda_{s r \alpha}^{s}}{d t}+\frac{R_{s r}}{L_{\varphi}} \lambda_{s r \alpha}^{s}\right)}{\lambda_{s r \beta}^{s}}=\frac{N_{1}}{D_{1}}
$$

Both (3) and (7) represent the base for an open-loop speed estimator, as proposed in [6]. The parameters $L_{\varphi} / R_{s r}$ (which is equal to the rotor time constant $\left.\tau_{r}=L_{r} / R_{r}\right), R_{s}, R_{s r}$ and $L_{t}$ are measured at motor terminals by conventional tests [8].

The weakness of (7) resides its sinusoidal numerator and denominator. In ideal conditions, their ratio would be always equal to the electromechanical speed. As a matter of fact, however, the concurrence of approximate parameter knowledge and digital rounding cause heavy estimation errors, as the denominator approaches to zero. This effect is depicted in Figure 2, which reports a simulation result of a digital openloop speed estimator based only on (7) and (3), during a ramp to one-eighth of its nominal speed. The simulated IM drive was a standard (sensored) Field Oriented Control (FOC), with feedback loop closed on measured speed, while the sensorless algorithm was running apart, tuned with the correct motor parameters.

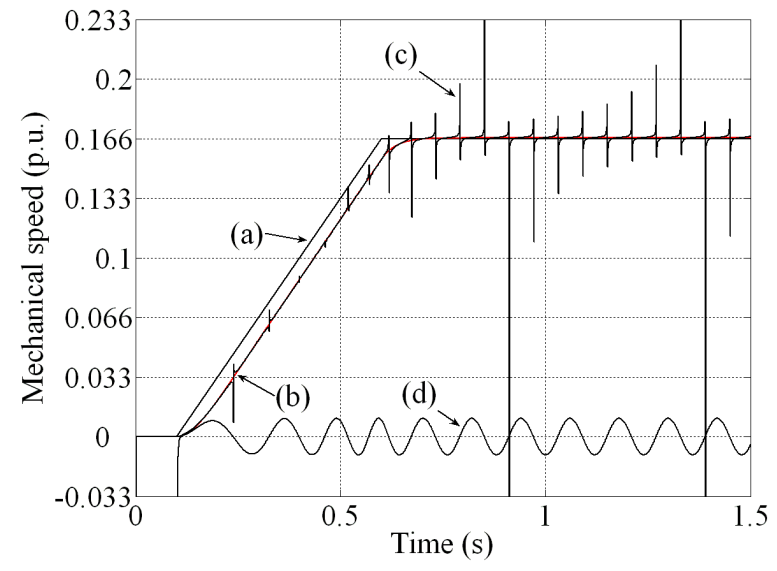

Figure 2. Open-loop speed estimator: (a) speed reference, (b) actual speed, (c) estimated speed based on (3) and (7), (d) estimated $\boldsymbol{\lambda}_{s r \beta}^{s}$

The bug is fixed by a hybrid mathematical model. Actually, the motor speed can be derived not only from (7), but also from the second of (6):

$$
\omega_{m e, 2}=\frac{\left(-R_{s r} i_{s \beta}^{s}+\frac{d \lambda_{s r \beta}^{s}}{d t}+\frac{R_{s r}}{L_{\varphi}} \lambda_{s r \beta}^{s}\right)}{\lambda_{s r \alpha}^{s}}=\frac{N_{2}}{D_{2}}
$$

Obviously, the estimate (8) suffers of the same limitations of (7). The only remarkable difference is that its denominator is computed from a law of cosines instead of sines, and then the zero-crossing instants of the denominators of (7) and (8) do not coincide. The proposed hybrid model is the weighted average of the two equations, as shown hereafter:

$$
\omega_{m e}=\frac{D_{1}^{2} \omega_{m e, 1}+D_{2}^{2} \omega_{m e, 2}}{D_{1}^{2}+D_{2}^{2}}
$$

The substitution of the last terms of (7) and (8) in (9) gives:

$$
\omega_{m e}=\frac{N_{1} D_{1}+N_{2} D_{2}}{D_{1}^{2}+D_{2}^{2}}
$$

The expression of the speed estimate is obtained by substituting (7) and (8) into (10), as follows:

$\omega_{m e}=\frac{R_{s r} i_{s \alpha}^{s} \lambda_{s r \beta}^{s}-\frac{d \lambda_{s r \alpha}^{s}}{d t} \lambda_{s r \beta}^{s}-R_{s r} \lambda_{s r \alpha}^{s} i_{s \beta}^{s}+\lambda_{s r \alpha}^{s} \frac{d \lambda_{s r \beta}^{s}}{d t}}{\lambda_{s r \alpha}^{s}{ }^{2}+\lambda_{s r \beta}^{s}{ }^{2}}$

As mentioned, the new mathematical formulation avoids every division by zero, since the denominator is the squared magnitude of the rotor flux linkage, referred to the stator. As a result, (3) and (11) represent an improved open-loop speed estimate, whose block scheme is outlined in Figure 3.

\section{SENSITIVITY ANALYSIS}

As anticipated, the proposed hybrid model for the speed estimate has improved parameter sensitivity with respect to that reported in [6]. A sensitivity analysis of (3) and (11) is carried out hereafter, with the aim of investigating the effects of parameters mismatch on the speed estimation.

Let us consider a generic real function $f(x, p)$, where $x$ is a variable (or a set of variables) and $p$ is a real positive 


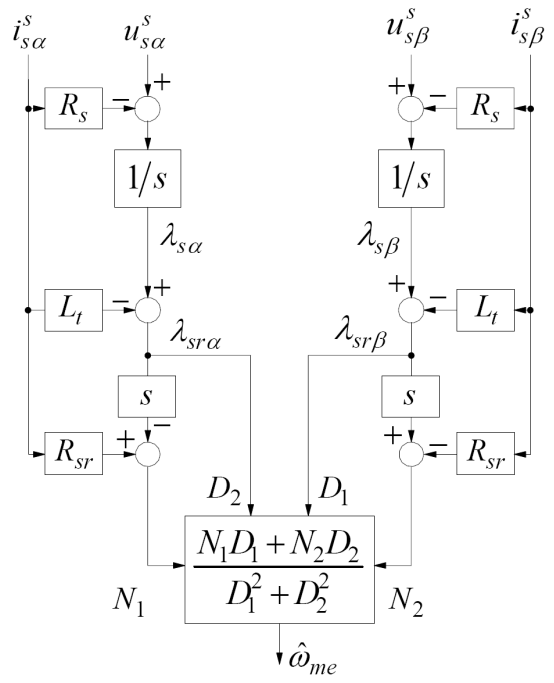

Figure 3. Open-loop speed estimator block scheme

parameter. At large, the sensitivity function is defined as the ratio between the relative variation of the function $f$, which is $d f / f$, with respect to the relative variation of $p$, which is $d p / p$. Effectively, the sensitivity is computed as the total derivative of $f$ with respect to $p$, multiplied by the factor $p / f$ :

$$
\tilde{S}_{f}^{p}(x, p)=\frac{\frac{d f}{f}}{\frac{d p}{p}}=\frac{d f}{d p} \frac{p}{f}
$$

The sensitivity analysis is a powerful tool during the process of selection of the best control algorithm. It is worth to note that (12) has an inherent singularity as the function $f$ gets near to zero. This fact, which is obvious in principle, does not depend upon the specific control algorithm, and it may alter a correct comparison of the sensitivities around $f=0$. The problem can be tackled by using a slightly different expression, in which the variations of $f$ and $p$ are related to their nominal values, instead of the actual ones:

$$
S_{f}^{p}=\frac{d f}{d p} \frac{p_{N}}{f_{N}}
$$

Clearly, a sensitivity function equal to one indicates that a percentage error on the parameter $p$ would be reflected to an equivalent percentage error on $f$. Consequently, the more the sensitivity function approaches zero, the less the function $f$ is influenced by an error on parameter $p$.

As a first example, the $S_{\omega_{m e, 1}}^{R_{s}}$ function for (3) and (7) have been obtained by applying the total derivative of (7) with respect to $R_{s}$. In details,

$$
\begin{aligned}
S_{\omega_{m e, 1}}^{R_{s}}= & \frac{d \omega_{m e, 1}}{d R_{s}} \frac{R_{s}}{\omega_{m e, 1}}=\frac{R_{s}}{\omega_{m e, 1}}\left(\frac{d \omega_{m e, 1}}{d \lambda_{s r \alpha}} \frac{d \lambda_{s r \alpha}}{d R_{s}}+\right. \\
& \left.+\frac{d \omega_{m e, 1}}{d \lambda_{s r \beta}} \frac{d \lambda_{s r \beta}}{d R_{s}}+\frac{d \omega_{m e, 1}}{d\left(\frac{d \lambda_{s r \alpha}}{d t}\right)} \frac{d\left(\frac{d \lambda_{s r \alpha}}{d t}\right)}{d R_{s}}\right)
\end{aligned}
$$

Introducing $I_{s}$ as the magnitude of the current vector, $\Lambda_{s r}$ as the magnitude of the rotor flux linkage referred to the stator, $\omega_{s}$ as the stator exciting frequency and $\varphi$ as the angle between $\lambda_{s}^{s}$ and $i_{s}^{s}$, the steady-state expression of $S_{\omega_{m e, 1}}^{R_{s}}$ gives:

$$
S_{\omega_{m e, 1}}^{R_{s}}=R_{s} R_{s r} I_{s} \frac{L_{\varphi} I_{s}\left[1+\cos \left(2 \omega_{s} t\right)\right]-2 \Lambda_{s r} \cos (\varphi)}{L_{\varphi} \omega_{m e, N} \omega_{s} \Lambda_{s r}^{2}\left[\cos \left(2 \omega_{s} t-2 \varphi\right)-1\right]}
$$

The function $S_{\omega_{m e, 1}}^{R_{s}}$ is time-dependant, so that a $R_{s}$ mismatch causes oscillations in the speed estimation. Similarly, it has been verified that also the sensitivity functions with respect to $R_{s r}, L_{t}$ and $L_{\varphi}$ are time-dependant. As a consequence, even small deviations in each parameter lead to oscillatory speed estimations. This is not the case of the proposed estimate, as reported in the next Sections.

\section{A. Stator resistance $R_{s}$}

The parameter $R_{s}$ appears only in (3), so that the sensitivity can be obtained by applying the total derivative of (11) with respect to $R_{s}$ :

$$
S_{\omega_{m e}}^{R_{s}}=\frac{R_{s} R_{s r} I_{s}^{2} \cos (2 \varphi)}{\omega_{s} \omega_{m e, N} \Lambda_{s r}^{2}}
$$

Opposite to (15), it does not depend upon time. As the speed approaches zero, the relative weight of the resistance over the inductive terms increasingly affects the estimate, while at high speed the influence of $R_{s}$ variations decreases to zero. This behaviour is reported in Figure 4, which shows $S_{\omega_{m e}}^{R_{s}}$ as function of $\omega_{s}$, for different load torque values.

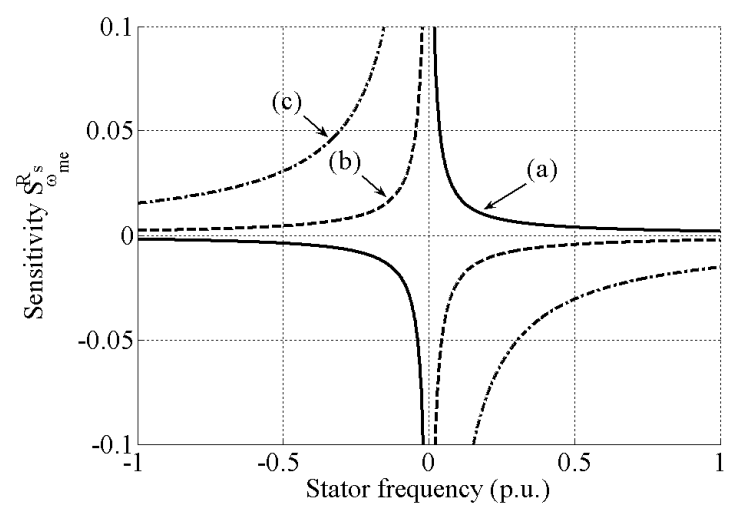

Figure 4. $S_{\omega_{m}}^{R_{s}}$ as function of $\omega_{s}$ : (a) no load, (b) $50 \%$ rated load, (c) $100 \%$ rated load

\section{B. Rotor resistance referred to the stator $R_{s r}$}

Since $R_{s r}$ appears only in (11), the steady state sensitivity function is simply:

$$
S_{\omega_{m e}}^{R_{s r}}=-\frac{R_{s r} I_{s} \sin (\varphi)}{\omega_{m e, N} \Lambda_{s r}}
$$

As expected, at no load $(\varphi=0)$ the sensitivity is null, since the steady state torque-producing component of the current $\left(I_{s r}\right)$, which flows through $R_{s r}$ (Figure 1), is null. Therefore, any change in the parameter shall not affect the estimated speed. 


\section{Transient inductance $L_{t}$}

Similarly to $R_{s}$, the transient inductance $L_{t}$ only appears in (3). The sensitivity function is then

$$
S_{\omega_{m e}}^{L_{t}}=-\frac{R_{s r} L_{t} I_{s}^{2} \sin (2 \varphi)}{\omega_{m e, N} \Lambda_{s r}^{2}}
$$

It is worth to note that both $S_{\omega_{m e}}^{R_{s r}}$ and $S_{\omega_{m e}}^{L_{t}}$ do not vary with speed, in the whole constant-torque region, while they are affected by flux-weakening operations. Increasing values of sensitivity are experienced for raising load torque, as reported in Figure 5, since both terms $I_{s}$ and $\varphi$ increase with load.

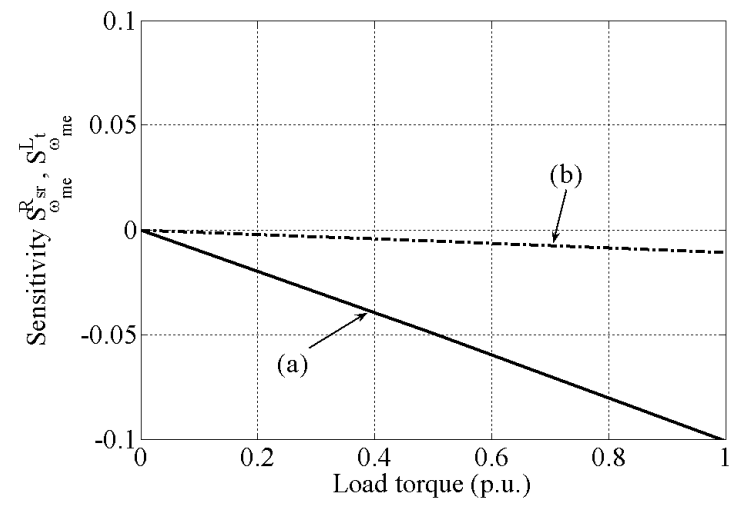

Figure 5. (a) $S_{\omega_{m e}}^{R_{s r}}$, (b) $S_{\omega_{m e}}^{L_{t}}$ as function of load torque.

\section{Magnetising inductance $L_{\varphi}$}

The magnetising inductance $L_{\varphi}$ does not appear neither in (3) nor in (11), as a feature of the adopted hybrid model expressed in (10). Consequently, the sensitivity function is null for all operating conditions:

$$
S_{\omega_{m e}}^{L_{\varphi}}=0
$$

\section{EXPERIMENTAL RESULTS}

The proposed sensorless scheme has been tested in several different working conditions, with the aid of a fast control prototyping (FCP) test bench, based on high speed microprocessor boards and a friendly user interface. The speed estimate has been used as feedback in a sensorless vector control of a $1 \mathrm{~kW}$ induction motor. The direct field oriented control [6] has been preferred to the indirect one, as the former does not use the estimated speed for the inner current control and thus it is, in principle, less sensitive to estimate errors. Different load torques have been generated by a hysteresis brake, coupled by a stiff joint to the motor shaft.

\section{A. The drift problem}

The flux linkage estimation represents a fundamental issue in either high-performance or sensorless drives. Nevertheless, the digital implementation of the integrator in (3) still represents a challenge [7], [9], [10]. Due to the drift and unknown initial value, a pure integration is unfeasible. In this work the solution reported in [10] was adopted, since its light computational requirements are consistent with the work scope. From the estimated flux linkage vector, the algorithm [10] provides an estimated stator current vector, which is compared with the measured one. The mismatch is multiplied by a proper gain and feed back to the integrator input, ensuring a rapid and effective integration correction.

\section{B. The dead-time effects}

Instead of measuring the stator voltages, which are necessary for (3), reference voltages $u_{s \alpha}^{*}, u_{s \beta}^{*}$ generated by the PI regulators of the FOC drive have been used. However, the substitution yields poor performances if a dead-time compensation is omitted. Figure 6 reports the real and estimated speed at steady-state in case of no compensation. As can be seen, a big steady-state error occurs, while a $6-t h$ harmonics effect is superimposed to the estimation outline. These well-known effects are described in [11].

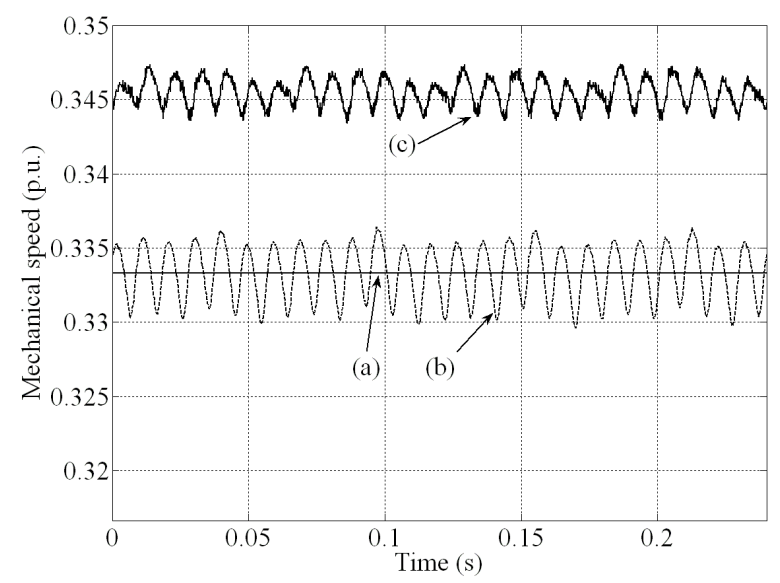

Figure 6. Effects of dead time on speed estimation: (a) speed reference, (b) estimated speed, (c) actual speed

In this paper, the repetitive control self-commissioning compensation procedure described in [11] has been adopted. The dead-time effects are greatly reduced, as shown in Figure 7. The availability of good (estimate) voltages turns out to be of great importance in extending the operational range of the sensorless algorithm at low speed, where dead-time effects are predominant.

\section{Dynamic behaviour}

Different speed reference profiles have been applied to the proposed estimator to evaluate its characteristics at various speed levels, both at no load and with applied load torques. Figure 8 shows a series of accelerations at no load, with different intermediate speed levels and speed ramps set to $2000 \mathrm{rpm} / \mathrm{s}$. The experiment is repeated in Figure 9, with a load torque of $50 \%$ of the rated torque. For the sake of completeness, Figure 10 shows a small-step speed response of the sensorless drive.

Figure 11 and 12 show the speed behaviour respectively at load onset and at detach, with a load torque value of $50 \%$ of the rated load. 


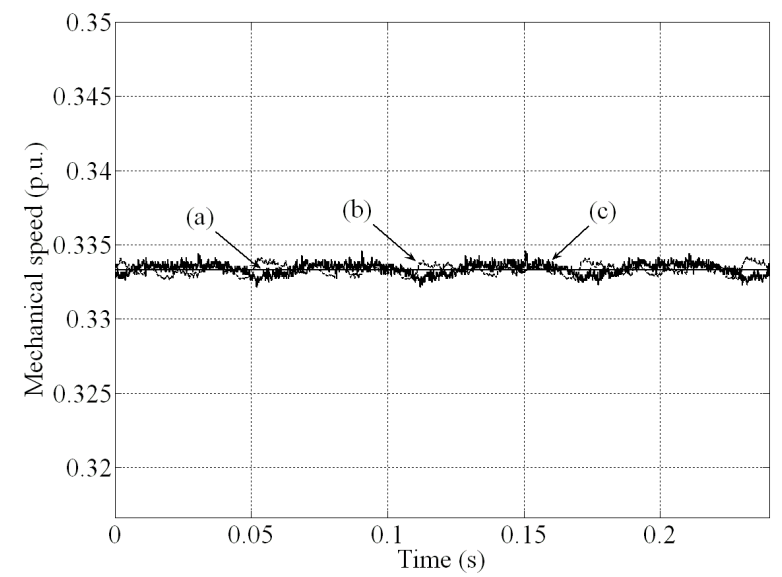

Figure 7. Speed estimation after dead time compensation: (a) speed reference, (b) estimated speed, (c) actual speed

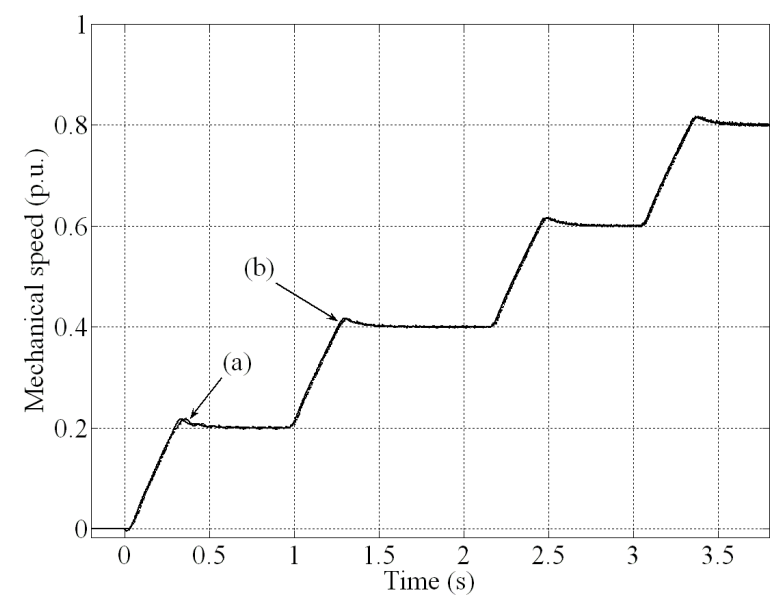

Figure 8. Speed rise (ramp of $2000 \mathrm{rpm} / \mathrm{s}$, no load): (a) estimated speed, (b) actual speed

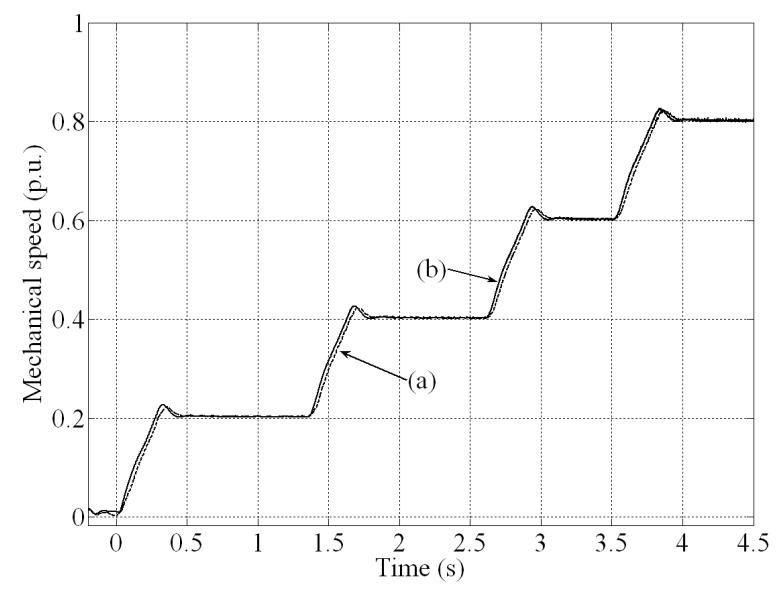

Figure 9. Speed rise (ramp of $2000 \mathrm{rpm} / \mathrm{s}, 50 \%$ of rated load): (a) estimated speed, (b) actual speed

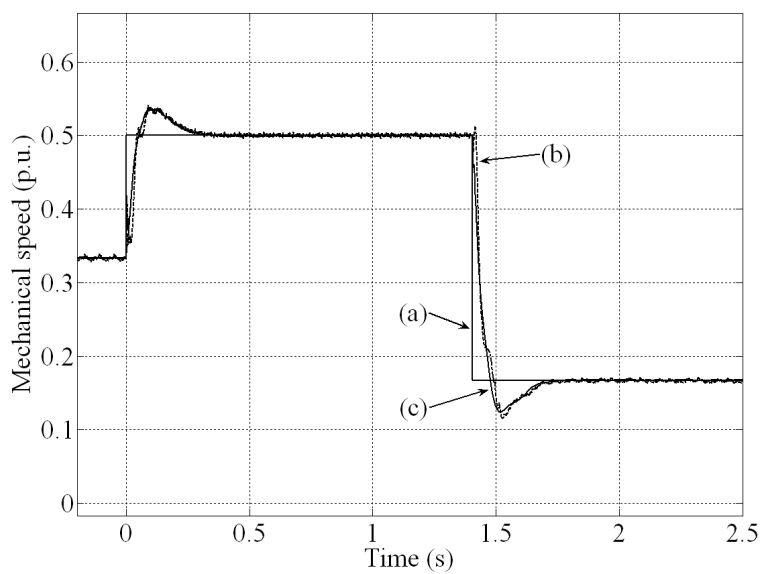

Figure 10. Speed step response: (a) reference speed, (b) estimated speed, (c) actual speed

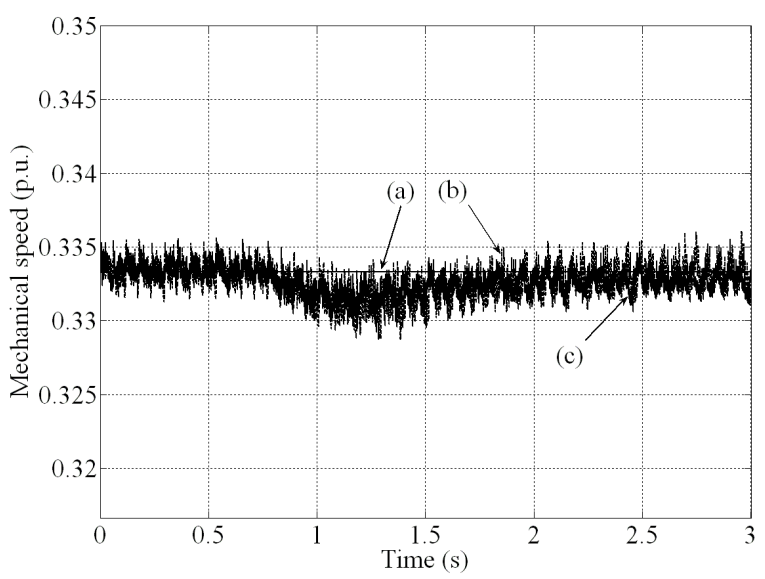

Figure 11. Speed profile at load onset (50\% of the rated load): (a) reference speed, (b) estimated speed, (c) actual speed

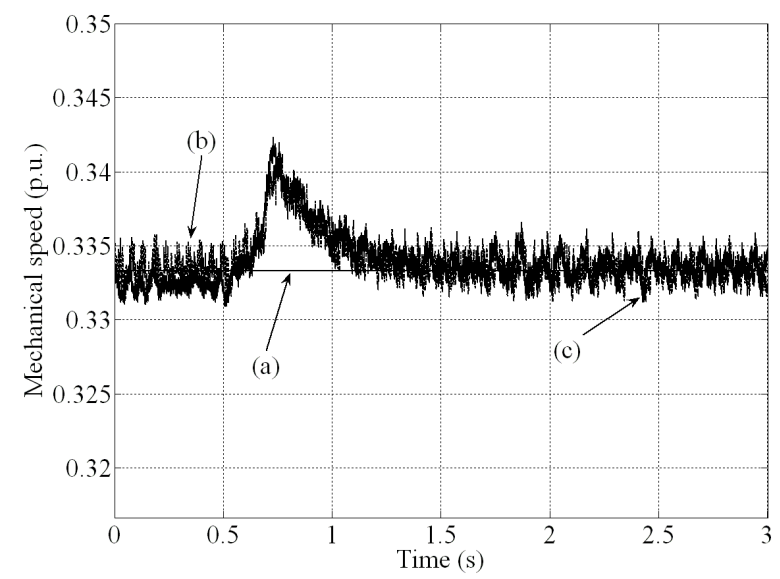

Figure 12. Speed profile at load detach (50\% of the rated load): (a) reference speed, (b) estimated speed, (c) actual speed 


\section{Parameter sensitivity}

An experimental sensitivity analysis has been performed on the proposed sensorless scheme. Waveforms have been obtained by forcing a step variation to each model parameter of the estimator. It is worth to note that the superimposition principle does not hold for every combination of parameter variations. In particular, it has been experienced that simultaneous variations of resistances (which is reasonable, since they both depend on temperature) produce a speed error lower than the arithmetic sum of (16) and (17). The effect, at time zero, of a simultaneous decrease of $40 \%$ of both $R_{s}$ and $R_{s r}$ values in the estimator is reported in Figure 13. The decrease of parameter values in the model simulates the temperature effect, which in fact increases the real motor resistances. The errors due to the single resistance variation should have been of $-0.26 \%$ of the nominal speed due to $R_{s}$ and of $0.03 \%$ due to $R_{s r}$. Actually, the global mean error is equal to $-0.055 \%$, which is less than expected, and far below the speed loop accuracy.

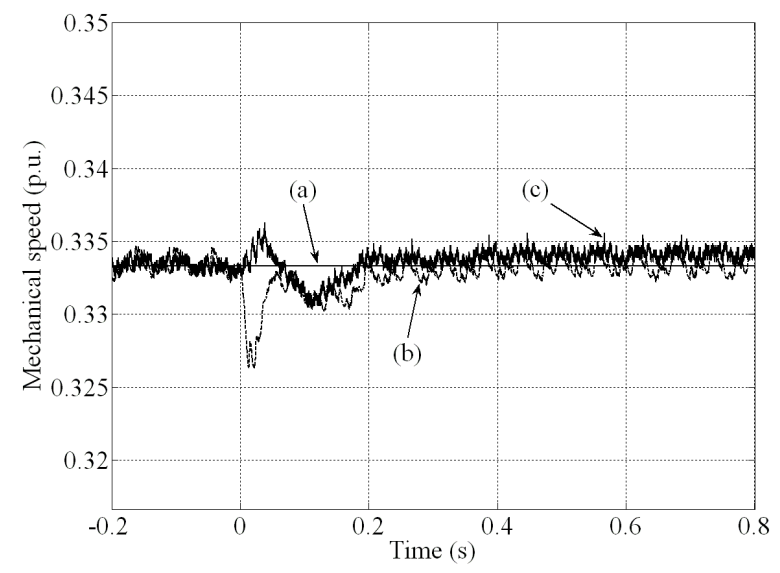

Figure 13. Speed profile with $-40 \%$ variation of $R_{s}$ and $R_{s r}$ : (a) reference speed, (b) estimated speed, (c) actual speed

The method is also quite insensitive to saturation effects, because of the intrinsic low values of (18) in almost the whole speed range, and also because $L_{t}$ represents a leakage inductance, normally unaffected by saturation.

Finally, a variation of $+40 \%$ of the $L_{\varphi}$ in the estimator (simulating the saturation effect) at time zero have been applied. On the strength of (19), no speed estimation error should arise, since $L_{\varphi}$ does not appear in (11). However, $L_{\varphi}$ is also a necessary parameter in the integral drift countermeasure mentioned in section IV-A, to obtain the estimated stator current from the estimated flux linkage. It follows that a variation of $L_{\varphi}$ indirectly influences the speed estimate, as shown in Figure 14, which reports a steady-state error of $0.22 \%$.

\section{CONCLUSIONS}

A novel open-loop speed estimation technique for lowcost sensorless IM drives has been presented and discussed in detail. First, an existing open-loop method, described in

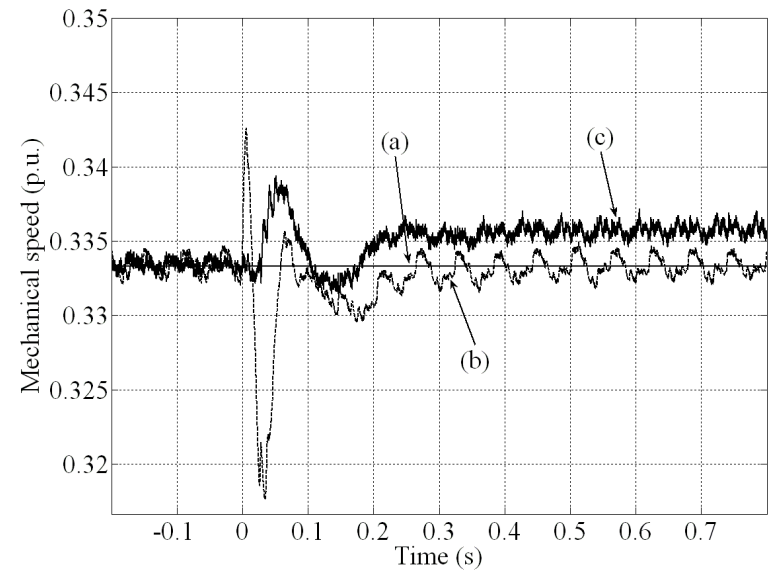

Figure 14. Speed profile with $+40 \%$ variation of $L_{\varphi}$ : (a) reference speed, (b) estimated speed, (c) actual speed

[6], has been analysed and tested. Then, the method has been improved by means of a hybrid mathematical model, which gives the system superior performances, in terms of both speed range and parameter sensitivity. An accurate mathematical analysis and an extended batch of experimental results are included in the paper, proving the robustness and the feasibility of the sensorless algorithm.

\section{REFERENCES}

[1] T.-S. Kwon, M.-H. Shin, D.-S. Hyun, Speed Sensorless Stator FluxOriented Control of Induction Motor in the Field Weakening Region Using Luenberger Observer, IEEE Transactions On Power Electronics, Vol. 20, No. 4, July 2005, pp. 864-869

[2] Y.-R. Kim, S.-K. Sul and M.-H. Park, Speed sensorless vector control of induction motor using extended Kalman filter, IEEE Transactions on Industry Applications, Vol. 30, No. 5, September/October 1994, pp. 1225-1233.

[3] S. Bolognani, L. Tubiana, M. Zigliotto, Extended Kalman filter tuning in sensorless PMSM drives, IEEE Transactions on Industry Applications, Vol. 39, No. 6, November/December 2003, pp. 1741-1747

[4] H. Kubota, K. Matsuse and T. Nakano, DSP-based speed adaptive flux observer of induction motor, IEEE Transaction On Industry Applications, Vol. 29, No. 2, March/April 1993, pp. 344-348.

[5] J. Maes, J. A. Melkebeek, Speed-Sensorless Direct Torque Control of Induction Motors Using an Adaptive Flux Observer, IEEE Transactions On Industry Applications, Vol. 36, No. 3, May/June 2000, p. 778-785

[6] P. Vas, Sensorless vector and direct torque control, Oxford University Press, 1998

[7] J. Holtz, J. Quan, Sensorless Vector Control of Induction Motors at Very Low Speed Using a Nonlinear Inverter Model and Parameter Identification, IEEE Transactions On Industry Applications, Vol. 38, No. 4, July/August 2002, pp. 1087-1095

[8] S. Bolognani, M. Zigliotto, Essentials of IM Parameters Measurement for FOC Drives Tuning, CD-ROM Proceedings of International Conference on Electrical Machines, ICEM'02, Bruges, Belgium, 2002.

[9] J. Hu, B. Wu, New Integration Algorithms for Estimating Motor Flux over a Wide Speed Range, IEEE Transactions On Power Electronics, Vol. 13, No. 5, September 1998, pp. 969-977

[10] R. Bojoi, P. Guglielmi, G. Pellegrino, Sensorless Direct Field Oriented Control of Three-Phase Induction Motor Drives for Low Cost Applications, Proceedings of IEEE/IAS 41st Annual Meeting, Tampa, Florida, October 2006, pp. 866-872

[11] S. Bolognani, L. Peretti, M. Zigliotto, Inverter Non-Idealities Override by Repetitive Control, IEEE Proceedings of the International Electric Machines and Drives Conference (IEMDC 2007), Antalya, Turkey, May 2007, pp. 71-76. 